\title{
Modeling cultural barriers in international trade
}

\author{
István Kónya* \\ Boston College ${ }^{\dagger}$
}

November, 2002

\begin{abstract}
The paper presents a model that analyses the role of cultural differences in international trade. The decision to study foreign cultures and languages is incorporated into a simple trade model, which captures some basic properties of cultural and language barriers. First, cultural costs differ from physical ones in that they can be eliminated by learning. Second, learning a language has economies of scale, thus smaller countries tend to invest more into learning. Third, learning decisions within one country impose an externality on trading partners, since learning by one party makes communication easier also for the other one. This implies that learning decisions are in general inefficient, and the paper derives the connection between the equilibrium and optimal outcomes. Finally, because of the substitutability of learning among countries, a policy where a country discourages learning - "cultural protectionism" - can be rationalized. Under certain conditions, such a policy can improve global welfare, not just that of the protecting country.
\end{abstract}

${ }^{*}$ I would like to thank Joseph Altonji, Gadi Barlevy and Kiminori Matsuyama for helpful comments and suggestions.

${ }^{\dagger}$ Correspondence: Economics Department, Boston College. Chestnut Hill, MA 02467. Tel: (617) 552-3690. E-mail: konya@bc.edu 


\section{Trade and culture}

International trade is not frictionless. Goods are costly to transfer, both because of geographical distance and cultural, language and institutional differences among countries. Even so, until the 1980's trade theorists tended to concentrate on an idealized world, where transporting goods across borders is costless. The rational for this was twofold. First, as with all imperfections, there are various possible ways in which trade is costly. To find the right model for frictions is thus a very difficult task. Second, in the traditional competitive models trade costs do not add much to the general understanding of specialization and other important questions.

The last two decades saw a marked change in this attitude, for both empirical and theoretical reasons. On the theoretical side, developments to incorporate imperfect competition and increasing returns to scale into trade models also led to the inclusion of trade costs. Starting with Krugman (1980), researchers used the "iceberg" form of trade costs together with a Dixit-Stiglitz type market structure (Dixit and Stiglitz 1977). This led to interesting new developments, not least to that of the "New Economic Geography" that sheds new light on the determination of the locations of economic activity. On the empirical side, the widely used and very successful "gravity model" of international trade ${ }^{1}$ incorporates various measures of physical and cultural trade costs among countries. Finally many empirical puzzles in international macroeconomics motivated Obstfeld and Rogoff (2000) to include trade costs into simple macro models.

In most of the theoretical developments physical and cultural impediments to trade were submerged into a single parameter. But there are reasons to try to examine the impact of geography and culture separately. First, geographical differences are not country specific, they apply to regions within countries as well. On the other hand, countries do have idiosyncratic cultural aspects, that separate them from other nations. Regional cultural variations within a country are usually much less pronounced then across borders. Thus arguably the interesting costs in international trade are the cultural ones, which differentiate international trade from interregional trade.

\footnotetext{
${ }^{1}$ The gravity model assumes that bilateral trade between two countries increases in their GDP's and/or populations, and decreases with the distance between them. Many models of trade can produce the gravity equation; indeed Deardorff (1995) argues that any meaningful trade model has to result in a version of it. For a recent contribution on the foundations of the gravity equation, see Anderson and van Wincoop (2001).
} 
There is some evidence that non-geographical costs are important. The empirical literature discovered that even controlling for distance, countries trade much less with each other than regions within a country do. Helliwell (1997) shows that OECD countries trade much less with each other then with themselves, after taking account of the usual gravity variables. Introducing language differences helps explain a large portion of the discrepancy, and much of the remaining part could be due to other cultural and institutional differences not accounted for. Thus in order to explain the "border effect", there is a clear need to look at cultural differences more closely.

Rauch (1999) also finds that language differences and historical ties are significant predictors of foreign trade. In a somewhat different context, Hall and Jones (1996) find that belonging to a major language group improves the economic performance of a country, even after controlling for a wide variety of factors. A possible explanation for this is that the knowledge of a widely used language enhances trade and the exchange of ideas. Finally, Helliwell (1997) presents some evidence that Canadian immigrants in US states and American ones in Canadian provinces enhance trade with their country of origin. An obvious explanation to this is that they possess knowledge of the culture and institutions of their native country, thus they can lower directly or indirectly the cultural cost of trade between the two countries.

Thus the evidence clearly points to the fact that cultural differences play an important role in international trade. Still, it is not clear whether distinguishing physical and cultural costs of trade adds much to theoretical understanding. Perhaps one could simply assume that the composite trade cost parameter takes a larger value across borders. There are, however, some interesting differences between cultural and geographical costs. When languages and cultures differ, it is necessary that someone learns the other's language and culture in order to communicate. It is true that physical trade costs can also be reduced by investment, but at any given time the state of technology limits how much these trade costs can be reduced. On the other hand, cultural barriers can be completely overcome, so the extent of learning is limited not by technological, but by market factors.

Also, in international transactions it is enough if one side learns the language and culture of the other. When a German firm exports goods into France, it is enough if the French partner speaks German or the German exporters speak French, but there is no need for both to learn. Thus in trade between different countries there is also the question of who invests into learning the other's culture. For physical costs of trade, often both 
countries have to invest in order to realize the gains: roads that only lead to the border but do not continue in the other country have limited benefits in international trade. Finally, gains from reducing physical trade costs seem more easy to appropriate, even if they benefit the other party. It is possible to charge for a use of a tunnel that connects two countries, but the potential to tax an English native because she can costlessly communicate with other parts of the world seems very limited.

In the light of these observations, this paper constructs a simple trade model with transportation costs that captures important properties of cultural barriers. The positive aspect of the model is to explain why some countries place so much emphasis on learning languages and knowing about other cultures, while others are very closed to foreign influence. The model is capable to generate this prediction, and it also sheds light on the effects of globalization on learning. In particular, cultural and physical trade costs influence learning differently: while a decrease in the latter normally encourages, cultural convergence discourages learning.

On the normative side, the paper compares the equilibrium outcome with the global planner's solution. It turns out that in general the equilibrium is inefficient, because learning has a positive external effect on the trading partner. Since learning in one country and another are strategic substitutes, there is a potential for "cultural protectionism": for a country to promote their own culture over foreign ones. The model reveals that a small tax on learning is always welfare enhancing in the protecting country, while it reduces welfare in the other nation. Interestingly such a policy might improve overall welfare, if the protecting country is large, cultures are very different and physical trade costs are small. Another reason why countries might want to promote their cultures is that multiple equilibria are possible, in which case countries can try to select the equilibrium that makes their language the one used in international communications.

There have been attempts to introduce cultural differences into models of international trade. A particularly interesting paper is Casella and Rauch (1998), who consider a search/network view of international trade. The idea in that paper is that imperfect information about foreign countries (caused partly by cultural differences) is the major impediment to trade, and information sharing networks can help overcome that barrier. The authors build an interesting model to examine the consequences of imperfect information and networks, and also consider some welfare implications. However, they do not consider the role of culture explicitly, and there is no learning involved. 
Belonging to information networks is exogenous, while the investment nature of acquiring information about foreign countries is incorporated into the model in this paper. Thus the approach here is much better suited to explore the strategic consequences of cultural costs.

Another effort to examine cultural differences is made in Lazear (1995). Though his model is built to examine the assimilation process of immigrants, it could easily be modified to model foreign trade. Cultural differences matter in Lazear's model because individuals are randomly matched to trade with each other, so communication difficulties result in less efficient trade. Since learning by one party eliminates the need to learn for the other (just as in this model), there is an externality involved with learning, resulting in suboptimal levels of investment. An important shortcoming in Lazear's model is that agents do not recognize the learning efforts of their partners, thus the model ignores the strategic nature of learning. This means, for example, that "cultural protectionism" cannot be used in Lazear's model. ${ }^{2}$

The rest of the paper is organized as follows. Section 2 introduces the basic building blocks of the model. Section 3 describes the equilibrium conditions, and explores the properties of the equilibrium. In section 4 the social planner's solution is derived, and compared with the equilibrium. Section 5 explores the potential for cultural protectionism. Finally, Section 6 concludes.

\section{The model}

This section constructs a very simple model of international trade, with differentiated goods but an exogenous number of varieties in countries. Although similar results can be derived from models where the range of varieties is endogenous, they do not yield analytical results as easily as the current approach. Also, the fixed number of varieties can be justified by referring to endogenous growth models, where the number of varieties in a country is

\footnotetext{
${ }^{2}$ As Lazear shows, a policy of "chauvinism" can work in his model, but it is quite different from what I call "cultural protectionism". There "chauvinism" means that immigrants into a country are forced to assimilate, and thus become natives. This in effect changes the ethnic balance between immigrants and natives, and thus changes incentives to learn even in the absence of the strategic link. In an international setting, this type of "chauvinism" is unlikely. Countries have limited scope to force other independent nations to acquire their culture. In my model, however, they can do so indirectly by recognizing the strategic connection between learning in different countries.
} 
proportional to the size of the country. Then the model in this paper can be viewed as a short-run snapshot of the innovating countries.

The world consists of two countries, 1 and 2, whose population levels are $L$ and $1-L$, respectively. Each person in a country is endowed with one unit of labor and $x_{0}$ units of an outside good. ${ }^{3}$ There is also a range of differentiated manufacturing goods, $c(i)$, with $i \in[0, N]$. Consumption of these goods solves the following optimization problem:

$$
\begin{gathered}
\max _{c(i)}\left\{x+\int_{0}^{N} \log c(i) d i\right\}, \\
\text { s.t. } \quad \int_{0}^{N} p(i) c(i)=w .
\end{gathered}
$$

The solution to this problem leads to the demand function for good i,

$$
c(i)=\frac{w}{N p(i)} .
$$

Each variety is produced by competitive firms. Production requires labor, and the unit input coefficient is normalized to one. The number of varieties produced in a country is predetermined, and it equals the labor size, $L$ in Home and $1-L$ in Foreign. Goods can be traded between countries, but only with a cost. To be more precise, transporting goods involves a physical $\operatorname{cost} \rho$ and a cultural $\operatorname{cost} \theta$. The costs are additive and incurred in the good itself. If one unit is required to arrive, $1+\rho+\theta$ units must be shipped from the country of origin.

Physical and cultural costs differ crucially in that the latter can be eliminated by learning. If a person decides to learn the culture of the other country, she only needs to incur the physical cost of importing goods. If she chooses not to learn, she might still be able to trade with someone in the other country who did learn. For simplicity, it is assumed that this occurs in proportion to the share of learners in the other country, i.e. the matching of trading partners is random. Let these shares be given by $\alpha$ in Home and $\beta$ in Foreign. An agent needs to forgo $c$ units of the outside good to learn. Populations are heterogenous in $c$, and the cost distribution is given by the c.d.f

\footnotetext{
${ }^{3}$ One way to interpret the outside good is that it is leisure that cannot be used for work. This is not completely unrealistic if all jobs require a fixed number of hours daily and there are no part-time or second jobs. In this case agents will work in the fixed hour jobs earning money to buy the other goods, and enjoy their leisure endowment $x_{0}$.
} 
$G(c)$ in both countries. For technical simplicity, it is assumed that $c \in[0, K]$ with full support, where $K \leq \infty$.

\section{Equilibrium}

From the assumptions above it follows that the supply of each variety is 1 . The equilibrium price of a good produced in country $\mathrm{j}$ equals the wage rate there, whereas the price paid in the other country is the home price times one plus the transportation costs. Since varieties are perfectly symmetric, there are only two different equilibrium conditions, one for goods produced in each country. Even these, however, are not independent: using Walras' Law one can be eliminated and one price can be normalized. Let $w_{1}=1$ and $w_{2}=w$, then the equilibrium condition for a good produced in Foreign can be written as

$$
1-L+\frac{(1+\rho)[\alpha+\beta(1-\alpha)] L}{w(1+\rho)}+\frac{(1+\rho+\theta)(1-\alpha)(1-\beta) L}{w(1+\rho+\theta)}=1,
$$

which yields

$$
w=1 .
$$

Agents will learn if the benefits from doing so are higher than the costs. The indirect utility function (excluding the cost of learning) in country $j$ for someone who bears the cultural cost is given by

$$
v^{l}=x_{0}-L_{k} \log (1+\rho+\theta),
$$

otherwise it is

$$
v^{n}=x_{0}-L_{k} \log (1+\rho) .
$$

If an agent learns, her utility is simply $v^{n}$. If she does not learn, her expected utility is the weighted average of $v^{l}$ and $v^{n}$, and the weights are the shares of learners and non-learners in the other country. The benefit from learning is the difference between these expected utilities. Everybody whose cost is smaller than the benefit will learn, otherwise they will not. This leads to the following cutoffs in the two countries:

$$
\begin{aligned}
& c_{1}=(1-L)(1-\beta) \log \left(1+\frac{\theta}{1+\rho}\right) \\
& c_{2}=L(1-\alpha) \log \left(1+\frac{\theta}{1+\rho}\right) .
\end{aligned}
$$


Using the notation $\mu=\log [1+\theta /(1+\rho)]$, the equilibrium conditions are written as

$$
\begin{aligned}
\alpha & =G[\mu(1-L)(1-\beta)] \\
\beta & =G[\mu L(1-\alpha)] .
\end{aligned}
$$

Notice that the proportion of learners in a country inversely depends on the proportion in the other. This gives a strategic aspect to the determination of the equilibrium, hence the term "the learning game" will be used when talking about this part of the model. With knowledge of the distribution function $G(c)$ one can solve for the equilibrium values of $\alpha$ and $\beta$. But even without specifying $G$, useful conclusions can be drawn, which are summarized in Proposition 1.

Proposition 1. If $G(c)$ is a continuous function there exists at least one stable equilibrium of the learning game. If the equilibrium is unique, the smaller country has a larger share of learners.

Proof. The existence of an equilibrium can be proved using Brower's FixedPoint Theorem. Let $\Gamma: \mathcal{R}^{2} \Rightarrow \mathcal{R}^{2}$ denote the function corresponding to the right-hand sides of (2). The continuity of $G$ ensures that $\Gamma$ is also continuous. Moreover, $\Gamma$ maps the unit square into itself, which is a compact and convex set. Thus there exist at least one fixed point of $\Gamma$, which is precisely the condition for the existence of an equilibrium.

To establish tâtonnement stability, the two equations in (2) can be treated as two reaction functions, $\alpha=R_{1}(\beta)$ and $\beta=R_{2}(\alpha)$. As Figure 1 illustrates, $R_{1}$ cuts the vertical axis above $R_{2}$, and $R_{2}$ cuts the horizontal axis to the left of $R_{1}$. These follow from the fact that $R_{1}(\beta)=0 \Rightarrow \beta=1, R_{2}(0)=$ $G(\mu L) \leq 1, R_{2}(\alpha)=0 \Rightarrow \alpha=1$ and $R_{1}(0)=G[\mu(1-L)] \leq 1$. Because of the continuity of $G(c)$ and hence $R_{i}$, there must be at least one intersection where $R_{1}$ cuts $R_{2}$ from above, which is the condition of stability.

Now imagine that the equilibrium is unique and that $L>1 / 2$ (country 1 is bigger). Start from a situation on $R_{2}$ where $\alpha=\beta$. Since $\mu(1-L)<\mu L$, it follows that $\alpha=R_{1}<R_{2}$. But a smaller $\alpha$ leads to an increase in $\beta=R_{2}$, and so on. This process continues until the unique equilibrium is reached, which is in this case globally stable. Since the starting situation was where $\alpha=\beta$, and throughout the adjustment process $\alpha$ decreases and $\beta$ increases, in equilibrium $\alpha<\beta$. 


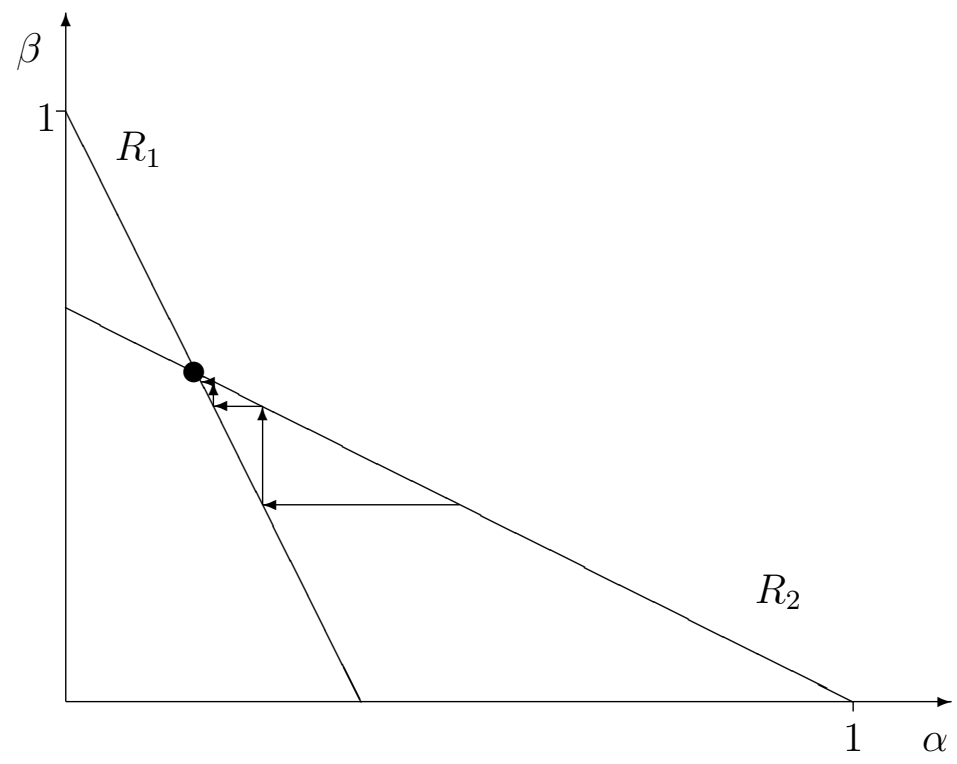

Figure 1: Equilibrium in the learning game

The last part of the theorem hints at some comparative statics results. As Appendix A shows, in any stable equilibria a marginal increase in one country's population will decrease the number of learners there and increase the number of learners in the other country. Graphically, an increase in $L$ corresponds on the picture to an inward shift of $R_{1}$ and an outward shift of $R_{2}$, which leads to an increase in $\beta$ and to a decrease in $\alpha$. The effect of an increase in $\mu$ is ambiguous. When $\mu$ rises, both lines shift out. Depending on the relative magnitude of the shifts, it is possible for either $\alpha$ or $\beta$ to decrease. In a "regular" situation both countries would have more learners. Since $\mu$ depends positively on $\theta$ and negatively on $\rho$, this implies that an increase in cultural costs encourages, while an increase in physical transportation costs discourages learning. Thus the effect of globalization depends crucially whether it involves mostly cultural or geographical convergence.

To get more clear results, a functional form needs to be specified for 
the c.d.f of learning costs. For simplicity, let $G(c)=c$, i.e. the uniform distribution on $[0,1]$. For interior solutions, the arguments of $G$ on the righthand sides of (2) must be below one. The condition for this is that $\mu<$ $\min \{1 / L, 1 /(1-L)\}$. Under such circumstances, the equilibrium learning shares are given by

$$
\begin{aligned}
& \alpha=\frac{\mu(1-L)(1-\mu L)}{1-\mu^{2} L(1-L)} \\
& \beta=\frac{\mu L[1-\mu(1-L)]}{1-\mu^{2} L(1-L)}
\end{aligned}
$$

It is easy to check that $\alpha<\beta$ iff $L>1 / 2, \alpha$ decreases with $L$ and $\beta$ increases with $L$. The effect of $\mu$ is also unambiguous, for example

$$
\frac{\partial \alpha}{\partial \mu}=\frac{(1-L)\left[1-2 \mu L+\mu^{2} L(1-L)\right]}{\left[1-\mu^{2} L(1-L)\right]^{2}},
$$

This expression is positive, unless $L>1 / 2$ and $[L-\sqrt{L(2 L-1)}] /[L(1-L)]<$ $\mu 1 / L$. Thus for $\alpha$ to fall with $\mu$, it must be that Home is bigger than Foreign, physical transportation costs are small and/or cultural differences are big.

This suggests an interesting pattern in cultural openness in large countries as physical trade costs fall. Since $\mu$ is inversely related to $\rho$, geographical globalization means an increase in $\mu$. According to the result above, in the early stages of the process ( $\mu$ small) globalization encourages learning. As trade costs fall, however, large countries will eventually reach a point when further cultural opening is not desirable. After this point, they actually start turning inward again, capitalizing on the smaller country's willingness to learn. Thus, given that the cultural difference from the outside world $(\theta)$ is substantial, the cultural openness of a large country will follow an inverted U-shaped pattern.

When $\min \{1 / L, 1 /(1-L)\}<\mu<\max \{1 / L, 1 /(1-L)\}$, everybody in the smaller country and nobody in the larger country learns. This result can arise when the two countries are very different in size, cultural costs are very high $(\theta$ big) and physical trade costs are low ( $\rho$ small). When $\mu>\max \{1 / L, 1 /(1-L)\}$, there are two stable equilibria, each with full learning in one country. Figure 2 illustrates the reaction functions in this case. Gains from learning are so high that when there is little learning in the other country, all people have an incentive to invest. Obviously it is more desirable for a nation that the other country incurs the costs, so policymakers 


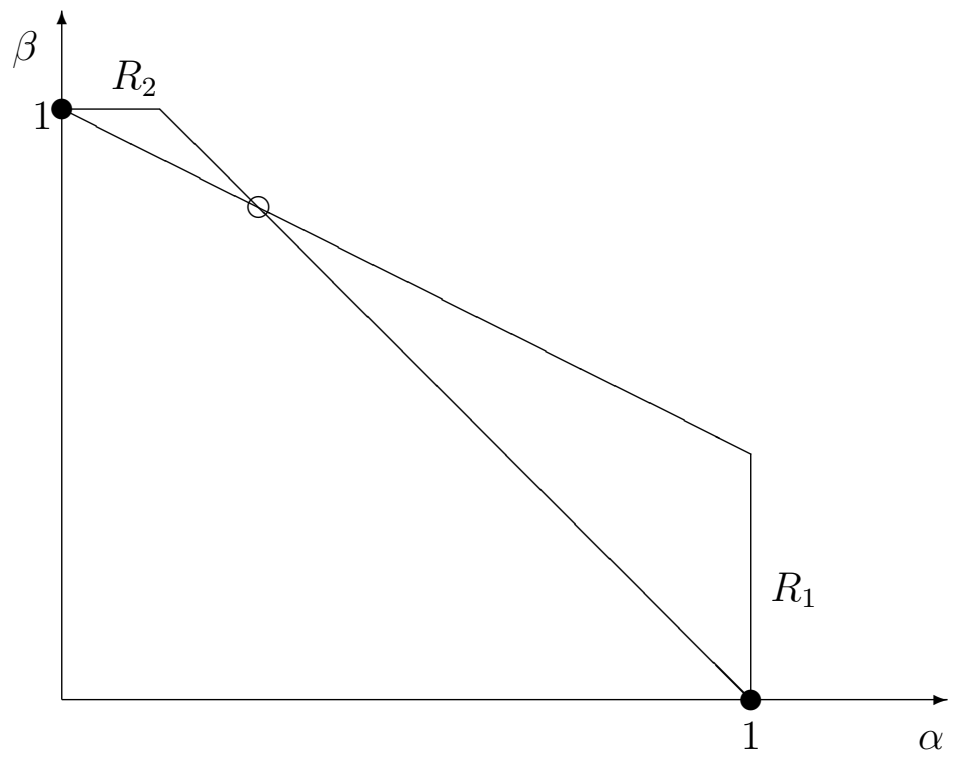

Figure 2: Multiple equilibria

have an incentive to promote their own culture. From a global point of view, however, it is the smaller country that should learn. Cultural trade costs are completely eliminated in both equilibria, but the total cost of learning is less when the smaller country invests.

To finish this section, it is instructive to look at how the total number of learners in the two countries,

$$
\alpha L+\beta(1-L)=\frac{\mu L(1-L)(2-\mu)}{\left[1-\mu^{2} L(1-L)\right]^{2}},
$$

depends on the parameters (in the case of an interior solution). It can be seen that the expression depends positively on $\mu$, as long as the solution is interior. The effect of $L$ is, however, ambiguous. In particular, the total number of learners is a concave function of $L$, and its maximum is achieved at $L=1 / 2$. Thus the most learning takes place when the two countries 
are of equal size. This is not surprising, given that the amount of trade is also highest in such a situation. In particular, the level of exports of Foreign, inclusive of transportation costs, are simply given by $L(1-L)$, the basic gravity equation. ${ }^{4}$ The level of imports in Home, not including transportation costs, is a complicated function of $L$ and the transportation $\operatorname{costs} \rho$ and $\theta$ :

$$
I M_{1}=\frac{L(1-L)}{1+\rho}\left\{1-\left(1-e^{-\mu}\right) \frac{(1-\mu L)[1-\mu(1-L)]}{\left[1-\mu^{2} L(1-L)\right]^{2}}\right\} .
$$

Although the second term in the square brackets is rather complex, it is still true that the largest volume of trade occurs when $L=1 / 2$. Thus the presence of cultural costs and learning modifies the functional form of the gravity equation, but it does not influence its main message. ${ }^{5}$

\section{Global welfare}

This section derives conditions for maximal global welfare. A very useful feature of the model is that consumers' utility is quasi-linear. This means that any point on the Pareto-frontier can be achieved by maximizing the sum of individual utilities and then redistributing the outside good endowment in a suitable way, which the government is assumed to be capable of. Let $v_{j}$ denote the sum of indirect utilities in country $j$, divided by the population level. The social planner chooses the optimal proportion of learners in the two countries, or alternatively, the cutoff levels that correspond to these proportions. Let $C_{j}$ stand for the optimal cutoff in country $j$. Ignoring the constant part of the outside good endowment $x_{0}$, per capita welfare in

\footnotetext{
${ }^{4}$ Transportation costs drop out because of the Cobb-Douglas sub-utility assumption.

${ }^{5}$ The effect of trade costs, however, does change with the inclusion of cultural costs. In particular, keeping $\rho$ constant, an increase in $\theta$ will actually increase trade for $L=1 / 2$ and $\mu$ above 1 . This nonlinearity should be taken into account when estimating the gravity model.
} 
country 1 and 2 can be written as follows:

$$
\begin{aligned}
v_{1} & =(1-L)\{[\alpha+(1-\alpha) \beta] \log (1+\rho)+(1-\alpha)(1-\beta) \log (1+\rho+\theta)\}- \\
& -\int_{0}^{C_{1}} c d G(c) \\
v_{2} & =L\{[\beta+(1-\beta) \alpha] \log (1+\rho)+(1-\alpha)(1-\beta) L \log (1+\rho+\theta)\}- \\
& -\int_{0}^{C_{2}} c d G(c) .
\end{aligned}
$$

The planner's problem is to maximize $v=L v_{1}+(1-L) v_{2}$, subject to the constraints that $\alpha=G\left(C_{1}\right.$ and $\beta=G\left(C_{2}\right)$.

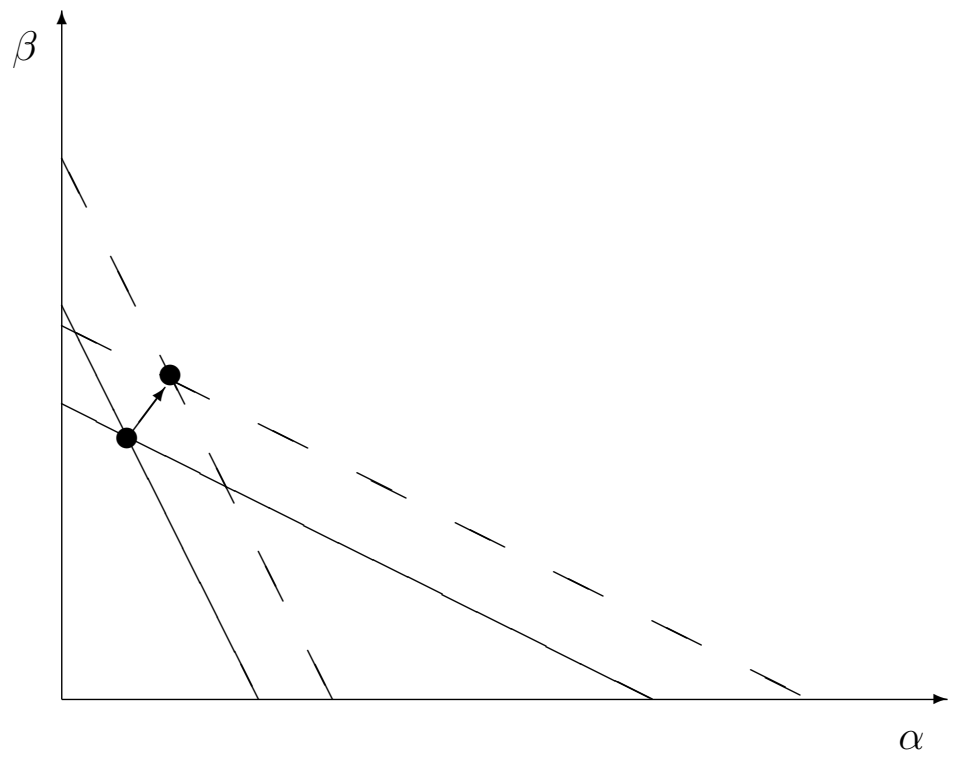

Figure 3: Equilibrium and optimum

The first-order conditions of the problem are simple, and can be written 
as

$$
\begin{aligned}
& C_{1}=2 \mu(1-L)(1-\beta) \\
& C_{2}=2 \mu L(1-\alpha)
\end{aligned}
$$

These imply that the solution to the global planner's problem is given by

$$
\begin{aligned}
\alpha & =G[2 \mu(1-L)(1-\beta)] \\
\beta & =G[2 \mu L(1-\alpha)]
\end{aligned}
$$

The structure of the optimal solution is very similar to the equilibrium one, but ceteris paribus the cutoffs are higher. This reflects the fact that learning has an external effect on the other country, since efficient matches only require one party to learn.

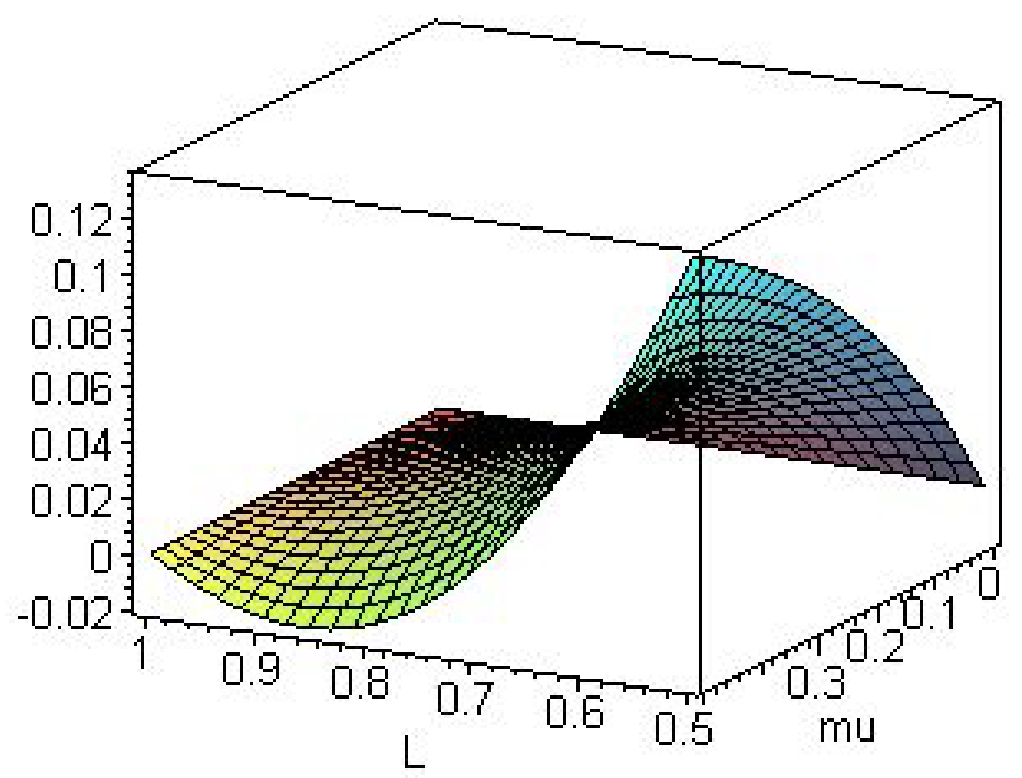

Figure 4: Equilibrium and optimum $\left(\alpha_{o p t}-\alpha_{e q}\right)$ 
In general, the optimal amount of learning in any country can be higher or lower than in equilibrium. In order to say more, it is useful to specify the cost distribution as before, $G(c)=c$. If the optimal solution is interior, it is given by

$$
\begin{aligned}
& \alpha=\frac{2 \mu(1-L)(1-2 \mu L)}{1-4 \mu^{2} L(1-L)} \\
& \beta=\frac{2 \mu L[1-2 \mu(1-L)]}{1-\mu^{2} L(1-L)},
\end{aligned}
$$

which requires the condition $\mu<\min \{1 /(2 L), 1 /[2(1-L)]\}$. Just as in equilibrium, $\alpha<\beta$ iff $L>1 / 2, \alpha$ decreases and $\beta$ increases with $L$. The effect of $\mu$ is again ambiguous: $\alpha$ decreases with $\mu$ when $L>1 / 2$ and $[L-$ $\sqrt{L(2 L-1)}] /[2 L(1-L)]<\mu<1 /(2 L)$.

The difference between the equilibrium and optimal solutions for $\alpha$ (in a range where both equilibrium and optimum are interior) is depicted on Figure 4. As the picture shows, there is more learning in equilibrium than in optimum when both $L$ and $\mu$ are large. This is a similar result to the one obtained above for $\mu$ : although equilibrium learning is too low in most countries, in large ones it might be too high if physical transportation costs are low and/or cultures in the two countries are very different. The total amount of learning in the two countries is given by

$$
L \alpha+(1-L) \beta=\frac{4 \mu L(1-L)(1-\mu)}{1-4 \mu^{2} L(1-L)},
$$

which is again increasing in $\mu$ for the relevant parameter range. As in equilibrium, the most learning takes place when $L=1 / 2$, the two countries are of equal size. In contrast to individual countries, the total amount of learning is always higher in optimum than in equilibrium.

To finish this section, the case when the optimum is not interior must be discussed. This occurs if $\mu>\min \{1 /(2 L), 1 /[2(1-L)]\}$. In contrast to the equilibrium, the optimum is always unique, because the solutions to the first-order conditions can be Pareto ranked. Thus in this range the optimal solution is full learning in the smaller country, and none in the larger one. The comparison between optimum and equilibrium is easy, but offers some interesting insights. First, the equilibrium can be optimal if it results in full learning for the small country. Second, it is possible that the equilibrium outcome (full learning) is optimal, but it takes place in the wrong country. 
Finally, in the range of an interior equilibrium and a full learning optimum, there is too little investment in the small country and too much in the large country.

\section{Cultural protectionism}

This section looks at the possible effects of a policy that might be called "cultural protectionism": Home makes it harder for its citizens to learn in order to force the other country to invest into its language. The policy tool the government uses is a simple tax $t$, that must be paid in addition to the learning cost $c$. Given the quasi-linearity of the utility functions, cultural protectionism is beneficial to a country if using it increases the sum of individual utilities. The government is assumed to give back the proceeds from the tax as lump-sum payments of the outside good.

The section will concentrate on a marginal deviation from a tax-free equilibrium. Per capita welfare in Home, $v_{1}$ from the previous section, can be rewritten to include the learning tax:

$$
\begin{aligned}
v_{1}(t) & =-(1-\alpha)(1-L)[\beta \log (1+\rho)+(1-\beta) \log (1+\rho+\theta)] \\
& +\int_{0}^{\mu(1-L)(1-\beta)-t}[-(1-L) \log (1+\rho)-c] d G(c) \\
& =-(1-L)[\beta \log (1+\rho)+(1-\beta) \log (1+\rho+\theta)]+\alpha t \\
& +\int_{0}^{\mu(1-L)(1-\beta)-t} G(c) d c .
\end{aligned}
$$

Taking the derivative with respect to $t$ and evaluating it at $t=0$ leads to

$$
\left.\frac{\partial v_{1}}{\partial t}\right|_{t=0}=\mu(1-L)(1-\alpha) \frac{\partial \beta}{\partial t}>0
$$

given that $\beta$ increases with $t$ (shown in Appendix B). Thus a small amount of cultural protectionism always helps the protecting country. This is completely analogous to the well-known result that a small tariff is always beneficial for a country that can influence its terms-of-trade.

The welfare change in country 2 can be analyzed similarly. Per capita welfare in Foreign, $v_{2}$, can be simplified to the following expression:

$$
v_{2}=-L[\alpha \log (1+\rho)+(1-\alpha) \log (1+\rho+\theta)]+\int_{0}^{\mu L(1-\alpha)} G(c) d c .
$$


Differentiation with respect to $t$ leads to

$$
\left.\frac{\partial v_{2}}{\partial t}\right|_{t=0}=\mu L(1-\beta) \frac{\partial \alpha}{\partial t}<0,
$$

since $\alpha_{t}<0$ (see Appendix B). Thus cultural protectionism always hurts the other country.

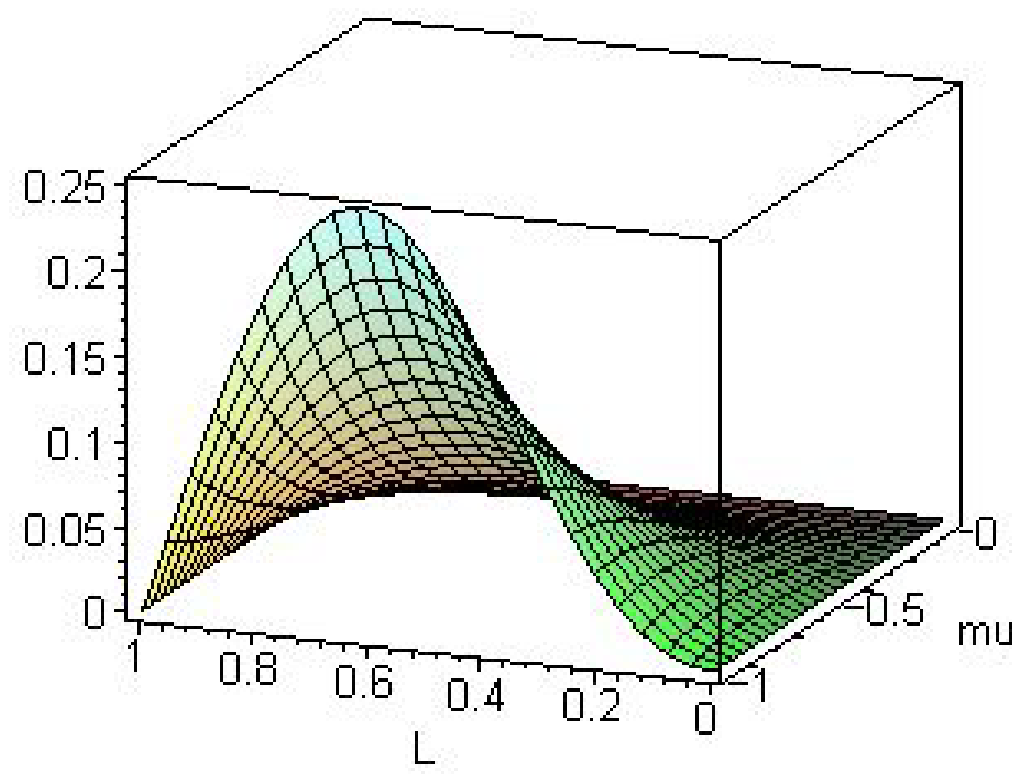

Figure 5: Gains from cultural protectionism

Although a small tax raises welfare for any country, it is interesting to ask what types of nations benefit most from such a policy. The general result in (6) is hard to interpret, so it is again useful to specify $G(c)=c$. In this case (6) takes the following form:

$$
\left.\frac{\partial v_{1}}{\partial t}\right|_{t=0}=\frac{\mu^{2} L(1-L)[1-\mu(1-L)]}{\left[1-\mu^{2} L(1-L)\right]^{2}} .
$$

Figure 5 plots the welfare increase against the parameters $L$ and $\mu$. As the picture shows, the gain is largest for large values of $\mu$, but for intermediate 
values of $L$ - although the peak is at a country size above $L=1 / 2$. This reflects two opposing forces. First, the most learning takes place at $L=1 / 2$, which means that there is much scope for shifting learning to the other country. On the other hand, larger countries have more power to protect, because the smaller nation has a bigger incentive to learn.

An interesting possibility is that cultural protectionism raises overall welfare. This might arise because the equilibrium outcome is not optimal. The Appendix derives that the effect of a small tax on world welfare is proportional to

$$
\mu g_{2} L(1-\alpha)-(1-\beta),
$$

where $g(\cdot)$ is the p.d.f of learning costs and $g_{2}=g[\mu L(1-\alpha)]$. Thus cultural protectionism might be beneficial if the protecting country is large and/or $\mu$ is large. This makes sense, since the previous section showed that under such circumstances there is too much learning in the large country, and too little in the small one (at least for the specific distributional assumption). Using $G(c)=c$ confirms these conjectures: cultural protectionism enhances world welfare when $L>1 / 2$ and $\mu>[L-\sqrt{L(2 L-1)}] /[L(1-L)]$.

\section{Conclusion}

This paper builds a model that incorporates cultural costs of international trade in addition to the usual physical ones. The are two features of cultural and language barriers that make them different from geographical costs: learning by individuals is essential to overcome them, but they do not capture the full benefits of their actions. Learning a language of another country also benefits the citizens of that nation, because they can communicate more efficiently without incurring the learning cost, an externality that is hard to internalize in many cases.

The paper derived the equilibrium conditions that determine the amount of learning in a two-country setting. The intuitive prediction of the model is that larger countries have fewer foreign language speakers. The effects of globalization depend crucially on the form it takes: a decrease in physical transportation costs is likely to facilitate learning, while cultural convergence has the opposite effect. The equilibrium is generally inefficient: there is too little learning overall, because individuals do not take into account the positive external effect of their learning decision. 
Finally, the possibility that a country can increase its welfare by discouraging learning was explored. The rational behind such an action is that learning is substitutable between countries, thus a nation can shift the cost of learning to the other country with a tax. The model shows that a small tax is always welfare enhancing for the protecting nation, but it hurts the other country. It is even possible that "cultural protectionism" increases overall welfare, when the protecting country is large, cultures are different and physical transportation costs are small. It was also shown that although all countries can benefit from cultural protectionism, it is large but not dominant countries that gain the most.

There are many possible extensions to the model. A particularly promising direction would be to include more than two countries. This would lead to much more complicated patterns of learning, and possibly multiple equilibria. 6 One could also study the question of a "dominant culture" (or in a more neutral phrasing, the emergence of a lingua franca). Another important question concerns the magnitude of the effects described in the paper, in particular the welfare gains attainable with cultural protectionism.

These extensions lead to many complexities, so they are left for further research. But even in its simple form, the model delivers interesting and perhaps unexpected results. Hopefully it convinces the reader that studying the role culture and language in the interaction and trade between countries is an important and fruitful enterprise.

\section{References}

Anderson, J. and van Wincoop, E. (2001). Borders, trade and welfare, in D. Rodrik and S. Collins (eds), Brookings Trade Forum, Washington: Brookings Institution.

Casella, A. and Rauch, J. E. (1998). Overcoming informational barriers to international resource allocation: prices and group ties, Working Paper 6628, NBER.

Deardorff, A. V. (1995). Determinants of bilateral trade: does gravity work in a neoclassical world?, Working Paper 5377, NBER.

\footnotetext{
${ }^{6}$ This may sound strange, since even in the two country framework multiple equilibria may arise. But with more than two countries, different types of equilibria would result, regardless of the functional form of $G$.
} 
Dixit, A. K. and Stiglitz, J. E. (1977). Monopolistic competition and optimum product diversity, American Economic Review 67: 297-308.

Hall, R. E. and Jones, C. I. (1996). The productivity of nations, Working Paper 5812, NBER.

Helliwell, J. F. (1997). National borders, trade and migration, Working Paper 602\%, NBER.

Krugman, P. (1980). Scale economies, product differentiation and the pattern of trade, American Economic Review 70: 950-959.

Lazear, E. P. (1995). Culture and language, Working Paper 5249, NBER.

Obstfeld, M. and Rogoff, K. (2000). The six major puzzles in international macroeconomics: is there a common cause?, NBER Macroeconomics Annual conference.

Rauch, J. E. (1999). Networks versus markets in international trade, Journal of International Economics 48: 7-35.

\section{Appendices}

\section{A Comparative statics}

The equilibrium conditions are given in (2) as

$$
\begin{aligned}
\alpha & =G[\mu(1-L)(1-\beta)] \\
\beta & =G[\mu L(1-\alpha)] .
\end{aligned}
$$

Taking the total differentials of these two equations lead to the following matrix equation:

$$
\left[\begin{array}{cc}
1 & \mu(1-L) g_{1} \\
\mu L g_{2} & 1
\end{array}\right]\left[\begin{array}{c}
d \alpha \\
d \beta
\end{array}\right]=\left[\begin{array}{c}
g_{1}(1-\beta)[-\mu d L+(1-L) d \mu] \\
g_{2}(1-\alpha)(\mu d L+L d \mu)
\end{array}\right]
$$

where $g_{1}=g[\mu(1-L)(1-\beta)], g_{2}=g[\mu L(1-\alpha)]$ and $g(\cdot)$ is the p.d.f of the learning cost $c$. The determinant of the left-hand side matrix is $\Delta=$ 
$1-\mu L(1-L) g_{1} g_{2}$, which is positive in a stable equilibrium (see the proof of Proposition 1).

To get the effect of a change in $L$ on the endogenous variables, set $d \mu=0$ and solve for $d \alpha$ and $d \beta$ :

$$
\begin{aligned}
& \frac{d \alpha}{d L}=-\frac{\mu g_{1}(1-\beta)+\mu^{2} g_{1} g_{2}(1-L)(1-\alpha)}{\Delta}<0 \\
& \frac{d \beta}{d L}=\frac{\mu g_{2}(1-\alpha)+\mu^{2} g_{1} g_{2} L(1-\beta)}{\Delta}>0 .
\end{aligned}
$$

For the effect of $\mu$, set $d L=0$ and again solve the equations for the endogenous variables:

$$
\begin{aligned}
& \frac{d \alpha}{d \mu}=\frac{g_{1}(1-L)(1-\beta)-\mu g_{1} g_{2} L(1-L)(1-\alpha)}{\Delta} \\
& \frac{d \beta}{d \mu}=\frac{g_{2} L(1-\alpha)-\mu g_{1} g_{2} L(1-L)(1-\beta)}{\Delta} .
\end{aligned}
$$

The sign of both of these expressions is ambiguous.

\section{B Cultural protectionism}

When country 1 taxes learning for its citizens, the equilibrium conditions change to the following:

$$
\begin{aligned}
\alpha & =G[\mu(1-L)(1-\beta)-t] \\
\beta & =G[\mu L(1-\alpha)] .
\end{aligned}
$$

Taking total differentials again and setting $t=0, d L=0$ and $d \mu=0$, the following matrix equation results:

$$
\left[\begin{array}{cc}
1 & \mu(1-L) g_{1} \\
\mu L g_{2} & 1
\end{array}\right]\left[\begin{array}{l}
d \alpha \\
d \beta
\end{array}\right]=\left[\begin{array}{c}
-g_{1} d t \\
0
\end{array}\right],
$$

The derivatives of $\beta$ and $\gamma$ with respect to $t$ (evaluated at $t=1$ ) are as follows:

$$
\begin{aligned}
& \alpha_{t}=-\frac{g_{1}}{\Delta}<0 \\
& \beta_{t}=\frac{\mu g_{1} g_{2} L}{\Delta}>0 .
\end{aligned}
$$


The effect on world welfare is given by

$$
\begin{aligned}
\frac{\partial\left[\left(L v_{1}+(1-L) v_{2}\right]\right.}{\partial t} & =\mu L(1-L)\left[(1-\alpha) \beta_{t}+(1-\beta) \alpha_{t}\right] \\
& =\frac{\mu g_{1} L(1-L)\left[\mu g_{2} L(1-\alpha)-(1-\beta)\right]}{\Delta}
\end{aligned}
$$

